\title{
Aortic bioprothesis dehiscence in the context of a chronic endocarditis
}

\author{
Inês Almeida ${ }^{1}$, Hélder Santos ${ }^{1}$, Hugo Miranda ${ }^{1}$, Mariana Santos $^{1}$, \\ Catarina Sousa ${ }^{1,2}$, Joana Chin ${ }^{1}$, Álvaro Laranjeira ${ }^{3}$ \\ ${ }^{1}$ Cardiology Department of Hospital Nossa Senhora do Rosário \\ - Centro Hospitalar Barreiro-Montijo, Barreiro, Portugal \\ ${ }^{2}$ Cardiovascular Center of the University of Lisbon, Lisbon School of Medicine, Lisbon, Portugal \\ ${ }^{3}$ Cardiac Surgery Department of Hospital de Santa Marta \\ - Centro Hospitalar Lisboa Central, Lisbon, Portugal
}

A 74-year-old woman carrier of an aortic bioprosthetic valve Trifecta St. Jude $21^{\circledR}$ implanted in 2013 in context of severe aortic stenosis was referred for a regular follow-up transthoracic echocardiogram. The patient was asymptomatic. Clinical examination revealed a systodiastolic murmur, grade IV/VI. Transthoracic echocardiogram showed a significant periprosthetic leak with rocking and expansion of the ascending aorta which was confirmed with transesophageal echocardiogram. It showed an aortic bioprosthetic valve with thickened leaflets, without images suggestive of vegetations, but there was evidence of an anechogenic free perivalvular space with flow inside and expansion during systole compatible with multiple pseudoaneurysms and a severe leak between 4 and 12 hours (Fig. 1A, B). The patient was proposed to undergo cardiac surgery. Blood cultures and microbiological exam of the excised valve were negative. During the procedure, an excision of the aortic bioprosthetic valve and the aortic root was made with enlarged surgical cleaning due to the presence of a false aneurysm down the prothesis suggestive of chronic endocarditis (Fig. 1C). A Freestyle ${ }^{\circledR}$ stentless porcine aortic root was successfully implanted. The post-operative period was uneventful.

Prosthetic valve endocarditis, $20-30 \%$ of all endocarditis', presents as dehiscence, fistula or pseudoaneurysm. A significant dehiscence of a prothesis with pseudoaneurysm formation is a rare complication that necessitates an urgent re-operation, given the high risk of spontaneous rupture, however it is particularly challenging due to the destruction of the aortic root and the need for complex repairs. The present case is notable as a complete asymptomatic presentation of pseudoaneurysm formation, demonstrating the role and importance of an specialized follow-up in these patients.

\section{Conflict of interest: None declared}

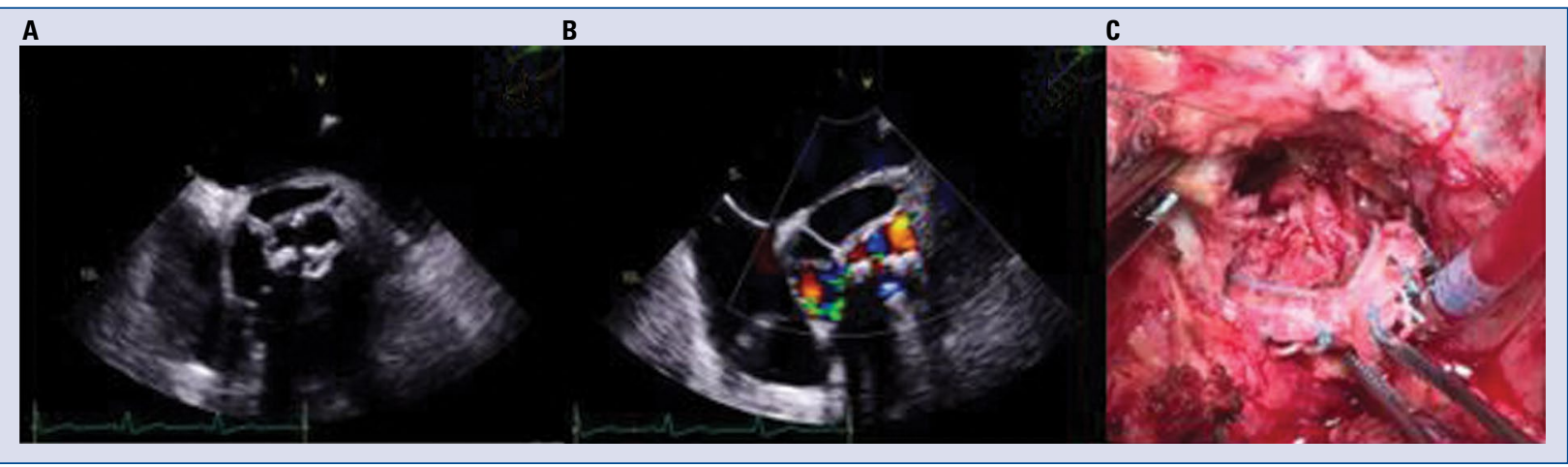

Figure 1. Transesophageal echocardiogram and surgical view of multiple pseudoaneurysms down the prothesis.

Address for correspondence: Dr. Inês Almeida, Avenida das Forças Armadas, 79C, 2834-003 Barreiro, Portugal, tel: +351 915186124, e-mail: inesalmeida91@gmail.com

Received: 29.02.2020 Accepted: 26.03.2020 